\title{
Educación inclusiva, profesores y estudiantes con discapacidad: una mirada desde lo relacional
}

\section{Inclusive education, teachers and students with disabilities: A look from the relational}

\author{
Rodolfo Cruz VAdillo*
}

Este trabajo forma parte de una investigación posdoctoral cuyo propósito fue analizar el tipo de relaciones que se constituyen en el proceso de inclusión de estudiantes con discapacidad. El artículo caracteriza las relaciones construidas a partir de la experiencia de la inclusión educativa de estudiantes con discapacidad desde la perspectiva de los profesores; realiza una aproximación al aspecto relacional en la experiencia de 41 profesores que han atendido a algún estudiante con discapacidad desde su ejercicio docente. La metodología fue cualitativa y se aplicó un cuestionario con siete preguntas abiertas; en el análisis se utilizaron algunos elementos de codificación y categorización propuestos por la teoría fundamentada. Los resultados permiten inferir que las relaciones se pueden pensar desde tres características: relaciones cuyo interés lo sustenta un sentido técnico; relaciones sustentadas en un interés con sentido práctico; y relaciones sustentadas con un interés con sentido transformador.

The present work is part of a post PhD research which main purpose was to analyze: what types of relationships are stablished in the process of inclusion of students with disabilities? The objective of this paper was "To characterize the relationships that have been built from the experience of educational inclusion of students with disabilities from the teachers' perspective", approached from the relational aspect in the experience of 41 teachers who have attended students with any disability during their teaching practice. The methodology was qualitative, the tool used was a questionnaire composed by seven open questions, the answers were analyzed, using some elements of coding and categorization proposed by the Grounded Theory. The results are about that relationships can be thought of from three characteristics: relationships whit an interest sustained by a technical sense, relationships based on an interest with a practical sense and relationships sustained with an interest with a transforming sense.
Palabras clave:

relación, educación inclusiva, profesores, estudiantes con discapacidad

\section{Keywords:}

relationship, inclusive education, teachers, students with disabilities

Recibido: 15 de febrero de 2019. | Aceptado para su publicación: 16 de julio de 2019. Recuperado de: https://sinectica.iteso.mx/index.php/SINECTICA/article/view/964 doi: 10.31391/S2007-7033(2019)0053-004

Sección: Investigaciones temáticas

* Doctor en Investigación Educativa. Profesor-investigador en la Universidad Popular Autónoma del Estado de Puebla. Líneas de investigación: política educativa y educación inclusiva. Correo: rodolfo.cruz@upaep.mx/ http://orcid. org/0000-0002-2561-1559 


\section{INTRODUCCIÓN: RUMBO AL PROBLEMA DE ESTUDIO}

$\mathrm{E}$ ste trabajo aborda las relaciones que se dan en el día a día en el espacio escolar que se considere "inclusivo" (Orón, 2018; Garro, 2017); hace visibles los intereses y sentidos técnicos e inmediatos y las cuestiones de enseñanza y aprendizaje (sistema microsocial), así como la realización de normas organizativas o universales que refieren al análisis de las políticas y los ordenamientos que organizan el espacio (sistemas meso- y microsocial); asimismo, desde esta perspectiva relacional y situada, se rescata ese espacio-tiempo de los sujetos en sus relaciones, al ubicarse en una posición inter alia entre los diversos sistemas y hacer visibles las formas en que lo macro-, lo meso- y lo micro- surgen en las interacciones cotidianas de convivencia. Estas relaciones se muestran a partir del juego sentido e interés, presente en la dinámica diaria de los profesores al trabajar con sus estudiantes.

Si bien la educación inclusiva intenta comprender una gran cantidad de grupos a los que históricamente se les ha negado la posibilidad de participar con equidad en los mismos espacios que la mayoría de la población, por ejemplo, personas de origen indígena, extranjeros o mujeres, en este trabajo nos interesa el proceso que se realiza solo con el colectivo de personas con discapacidad. El espacio concreto es la institución escolar y el objeto de estudio son las relaciones que se constituyen en el proceso inclusivo y que, en su ejercicio e iteración diaria, pueden permitir visibilizar los planos jurídico-políticos (macro-), organizacionales (meso-) y de aprendizaje áulico (micro-) (Michailakis \& Reich, 2009).

Este texto es fruto de una investigación posdoctoral cuyo propósito central fue analizar el tipo de relaciones que se constituyen en el proceso de inclusión de estudiantes con discapacidad. Para ello, buscamos responder las siguientes preguntas: ¿qué características presentan las relaciones construidas en el proceso de inclusión de estudiantes con discapacidad? ¿Qué elementos de esas relaciones pueden posibilitar el proceso de inclusión, o no? El objetivo es caracterizar las relaciones que se han construido a partir de la experiencia de la inclusión educativa de estudiantes con discapacidad desde la perspectiva de los profesores. Para lograrlo, presentamos una primera aproximación al análisis de las relaciones que se han construido en torno al proceso inclusivo, las cuales, en un primer momento, pueden ser materializadas desde un plano discursivo, y rescatan las ideas y experiencias de los profesores a partir del establecimiento de un tipo de relación con algún estudiante con discapacidad.

En la revisión de la literatura hemos observado que el tema de lo relacional ha quedado más o menos desatendido, puesto que, por lo regular, los estudios se han interesado por objetos como las prácticas de enseñanza y aprendizaje o las leyes y políticas sobre inclusión. Este texto tiene que ver con aquellos objetos que poseen menor materialidad concreta y que, sin embargo, sostienen las prácticas a nivel técnico-pedagógico y responden a una visión política e institucional.

La justificación para esta investigación tiene que ver, por un lado, con un vacío en la literatura sobre el papel de lo relacional en el proceso inclusivo, que está más bien centrado en los conceptos, los significados, los instrumentos, las técnicas, los métodos y no en aquello que los sostiene, es decir, las relaciones que se establecen en el encuentro cotidiano. 
Por otro lado, puede coadyuvar, desde una perspectiva política y educativa, a la comprensión de los procesos in situ de inclusión, al presentar las rupturas o continuidades, los posibilitadores y obstaculizadores que, desde el plano jurídico- normativo hasta el práctico-educativo, se constituyen cuando los elementos histórico-sociales se encuentran y las identidades se relacionan. Estas identidades son producto de ese conjunto de interacciones e intercambios simbólicos que se dan en lo social (Goffman, 2001). En este sentido, la identidad juega un papel central en el proceso inclusivo, pues permite pensarlo desde el plano del reconocimiento del otro que es considerado diferente, característica que supone más bien riqueza que déficit.

\section{EDUCACIÓN INCLUSIVA: DIVERSAS FORMAS DE PROBLEMATIZACIÓN}

El interés por hacer de la escuela un espacio inclusivo es una preocupación compartida desde varias latitudes; esto es visible en diversos tratados y políticas que han intentado dar un ordenamiento al tema educativo y escolar. En este marco se han presentado declaraciones, tratados y convenios con la finalidad de emplazar a las naciones hacia una educación para todos e inclusiva. Algunos ejemplos de estos esfuerzos pueden encontrarse en documentos como la Declaración de Conchabamba y Recomendación (Unesco, 2001); Temario abierto sobre educación inclusiva (Unesco, 2004); la Declaración de Salamanca y la Conferencia Mundial sobre Necesidades Educativas Especiales: Acceso y calidad (ONU, 1994); la Declaración Mundial de Educación para Todos y el Marco de Acción para Satisfacer las Necesidades de Aprendizaje (Unesco, 1990); La educación inclusiva: el camino hacia el futuro (ONU, 2008), el Foro Mundial para la Educación (Unesco, 2000), entre otros.

La preocupación y ocupación por el tema no solo ha sido a nivel político, incluso ha superado el interés de educadores y especialistas en el campo educativo. Cuando se revisa la literatura, encontramos una gran cantidad de disciplinas pertenecientes a las humanidades y las ciencias sociales, que, desde diversas lógicas, han realizado interesantes abordajes. Si bien hay trabajos dirigidos al aula, existen investigaciones que plantean lo complejo del proceso inclusivo y reconocen que su estructura supera el espacio de la escuela, al centrarse en un proceso más social (Michailakis \& Reich, 2009).

Por un lado, hay escritos que versan sobre las competencias docentes y los resultados de aprendizaje como elementos finales del proceso inclusivo (sistema microsocial) (Haug, 2016; Adetoro, 2014), cuyo interés central tiene que ver con los aspectos que hacen a un docente eficaz, con competencias de enseñanza que permitan a los estudiantes aprender los contenidos escolares. Otros trabajos dan cuenta de los aprendizajes per se (Fitch, 2003; Ferguson, 2014), posibilitados a partir de las experiencias inclusivas dentro de las escuelas.

Por otro lado, tenemos investigaciones que tratan las políticas "inclusivas" y algunas prácticas en torno a ellas (sistema macrosocial) (Kiyuba \& Tukur, 2014; Mitchell, 2015). En ese plano político se muestra la necesidad de indagar sobre la incorporación de ordenamientos sustentados en los derechos humanos como una de las vías para lograr reducir las exclusiones (Mukherjee \& Bear, 2017). Otros trabajos manifiestan su preocupación por las posibilidades de alineación entre los diferentes niveles estructurales, es decir, refieren la necesidad de un esfuerzo de articulación entre lo maso-, meso- y microsocial, donde los medios disponibles de la política están en relación 
directa con las propias prácticas y estructuras organizativas (Miles \& Singal, 2008; Chakraborti-Ghosh, 2017; Douglas, McLinden, Robertson, Travers \& Smith, 2016).

Nuestro trabajo se asocia más a este último grupo de investigaciones; sin embargo, si bien nos interesa la visión holística, no nos enfocamos en la alineación de los sistemas; su espacio es la práctica cotidiana donde se pueden materializar, a partir de las relaciones construidas, las visiones sobre el proceso y las formas en que esos sistemas se hacen presentes con los encuentros y desencuentros del día a día (Ngcobo \& Muthukrishna, 2011).

\section{EDUCACIÓN INCLUSIVA: DESDE UNA PERSPECTIVA RELACIONAL}

La educación inclusiva no solo es una cuestión de derechos o de aprendizaje; representa también una apuesta ontoepistemológica para desmontar un conjunto de representaciones que parecen ubicar a las personas con discapacidad (y otros colectivos) en un espacio y una posición en el campo social que se encuentra siempre al margen de los considerados "normales".

En el caso de las personas con discapacidad, la historia de ese aspecto educativo se puede remontar a todo el movimiento de educación especial, movimiento escolar y educativo en el cual las relaciones entre los profesores y los estudiantes con discapacidad lejos estaban de representar un espacio dialógico y horizontal. El saber especializado por sobre el objeto de conocimiento implicaba una mirada en la que la relación saber-poder estaba centrada en el interés y sentido de la patologización y psiquiatrización de los sujetos (De la Vega, 2010; Thomas \& Loxley, 2007; Skrtic, 1996). La idea era realizar un tipo de ortopedia sobre los cuerpos no considerados válidos (Pié, 2015), necesitados de un proceso pedagógico, en el cual la modificación conductual, cognitiva, moral y disciplinar eran la finalidad primordial para poder incorporar al sujeto a la vida social. En cierto sentido, la necesidad de normalizar al otro, de establecer un tipo de poder que constituya sujetos "normales" (Foucault, 1999, 2005).

Los espacios así estaban dispuestos como exclusivos de determinadas identidades, subjetividades, que en las relaciones asimétricas entre el saber especializado de los profesores y el propio estudiante con discapacidad eran reelaboradas día a día desde la visión del déficit (Brogna, 2009). Un conocimiento que situaba, clasificaba, nombraba y, a su vez, estigmatizaba (Goffman, 2001) al efectuar las asignaciones de sentido y sujeciones desde el aparato escolar (Popkewitz y Brennan, 2000).

El proceso de escolarización no solo implica la articulación y disposición de una serie de estrategias de aprendizaje y enseñanza o de un conjunto de políticas y ordenamientos, tiene que ver también con un elemento ontoepistemológico (Ocampo, 2015) en el cual el sentido, el lugar asignado y la identidad construida parecen ser nodales en esa empresa. Este ejercicio, sin duda, señala las relaciones constituidas y mediante las que se puede materializar un tipo de existencia en lo social.

En México, desde 1993, gracias a visiones internacionales, ordenamientos y políticas, ya expuestas, se dio un nuevo movimiento que buscaba reconfigurar las formas y lógicas que dirigían el proceso educativo de los estudiantes con discapacidad. Este movimiento, conocido como la integración educativa, pretendía incorporar y, por ende, construir nuevas relaciones hacia los estudiantes con alguna necesidad educativa especial 
asociadas a alguna discapacidad o con aptitudes sobresalientes a la escuela regular, puesto que la tradicional estructura de la educación especial empezó a verse como altamente segregatoria y excluyente (Adame, Jacobo y Alvarado, 2016; García, 2018).

A pesar de los esfuerzos serios por cambiar las formas de participación de lo escolar, tal empresa no logró su cometido; por ejemplo, el papel del profesor no superó la visión biomédica y el interés por "reparar". En esa política se enseñó al docente el requisito de identificación y diagnóstico de necesidades educativas especiales, acciones supeditadas a criterios todavía de normalidad y, sobre todo, a la representación propia que este agente tenía del estudiante, al corresponderle, como tarea central, la asignación de la "necesidad", y el sentido y posición del sujeto en el espacio escolar (Cruz, 2013).

Tampoco las categorías establecidas ayudaron, puesto que el propio concepto de necesidad resultaba problemático al ubicarse desde una visión esencialista sobre el sujeto y al equiparar la escolarización con una cuestión de primer orden, que naturaliza los déficits de aprendizaje como cuestiones meramente inherentes a la persona (Thomas y Loxley, 2007). Al final, no quedaba claro si la necesidad era del estudiante en su ser o de una escuela y de unos profesores que tenían la tarea de moralizar, disciplinar y normalizar a esos estudiantes que salían de lo común (Popkewitz y Brennan, 2000; Foucault, 1999, 2005), lo que constituía relaciones de poder-saber asimétricas: un sujeto que sabe sobre el otro y le aplica una determinada voluntad de verdad, un poder disciplinar que constituía una identidad "frágil" (Varela y Álvarez, 1989).

Desde la perspectiva de la educación inclusiva, el espacio paradigmático y relacional parece plantearse diferente, pues pasa de un tipo de ciencia biológica a una social, en el que lo anormal, lo desviado, es más un constructo social que un elemento intrínseco al sujeto. Hablar hoy de educación inclusiva implica, por un lado, un ejercicio deconstructivo que supere las visiones esencialistas de la educación especial (Thomas y Loxley, 2007) y, por otro, tensionar las nociones de normalidad que todavía circulan de forma constante y que constituyen las lógicas de organización del espacio, así como los lugares de asignación y relación en lo escolar (Vlachou, 2007).

Si bien la educación inclusiva está vinculada al espacio institucional de la escuela, su reorganización no es solo material, sino también relacional; la participación y colaboración parecen estar en el centro de los principios que pueden guiar el actuar de los profesores (Ainscow, 2014), y posibilitan el replanteamiento de la propia institución escolar, por ejemplo, el papel del currículo en los procesos de inclusión cuyo principio de flexibilidad puede permitir el encuentro de las identidades y el enriquecimiento relacional de ellas.

La educación inclusiva refiere una verdadera experiencia educativa (Contreras y Pérez, 2010) que es beneficiosa para el estudiante y también para el profesor; un proceso de implicaciones mutuas, en el cual la experiencia es relación, y la relación es la que hace factible un saber y la posibilidad de crecimiento desde la práctica y para la práctica humana. Del mismo modo, es una alfabetización ética (Echeita, 2014), una oportunidad para humanizarnos (López, 2011), una filosofía que puede servir de puente para la constitución de un encuentro con el otro; una comprensión empática, un reconocimiento, una apertura que tiene como medio el aprendizaje (Giné, 2009) y como fin la construcción de una identidad que permita poder pensarse como parte de algo; nunca más en sus márgenes ni en el centro de las miradas "especializadas". 
Por otra parte, es un reconocimiento y visibilización de relaciones sociales que producen exclusión (Slee, 2012; Stainback y Stainback, 2011), que entorpecen el encuentro intersubjetivo, la participación, la idea de pertenencia y, por ende, una identidad que se reconoce con otras identidades con las cuales convive. Para este trabajo, la educación inclusiva son las relaciones que se establecen en el día a día y que posibilitan o imposibilitan los encuentros, la construcción de las identidades y las formas de existencia en lo social.

En línea con lo anterior, lo relacional tiene que ver con dos premisas: "1) la realidad se compone de relaciones, es en sí misma relacional; 2) lo que hay que observar son las relaciones y entender por qué se dan esas y no otras y qué procesos pueden modificarlas para hacerlas más humanas..." (Garro, 2017, p. 638). Estas premisas señalan la imposibilidad de seguir abordando lo social únicamente como el juego de sistema o ambiente o estructura o acción para mirar las relaciones que sostienen y, a su vez, son sostenidas por esos sistemas. Así “... el saber, la comprensión y la explicación del cambio social dependen de la acción recíproca entre estados subjetivos y motivaciones individuales, además de códigos simbólicos, culturales y normativos y condicionantes estructurales" (Garro, 2017, p. 639).

Desde esta perspectiva, el centro está en las valoraciones, mirada axiológica que supera el mero carácter instrumental-técnico de la racionalidad humana, y da cuenta de la importancia de las relaciones sociales que se entablan en lo social. Estos valores están íntimamente asociados a las actitudes, emociones y sentimientos que se presentan en la relación (Orón, 2018), así como las visiones en torno a determinado acto, por ejemplo, las ideas que se construyen en la acción y que pueden ser valoradas por los agentes que las vivieron.

De igual modo, las relaciones tienen que ver con los sentidos e intereses de los propios participantes; cuando se incluye a un estudiante, la pregunta por el sentido es primaria: ¿qué sentido tiene que él esté aquí? Por otra parte, se refiere a los intereses o finalidades, ¿para qué este estudiante con discapacidad debe educarse? Las respuestas pueden ser diversas, como diversos son los sentidos e intereses que se juegan en una relación. En el esquema 1 se ilustra la relación que acaba de señalarse. 
Esquema 1. Plano relacional: aproximación al estudio de las relaciones

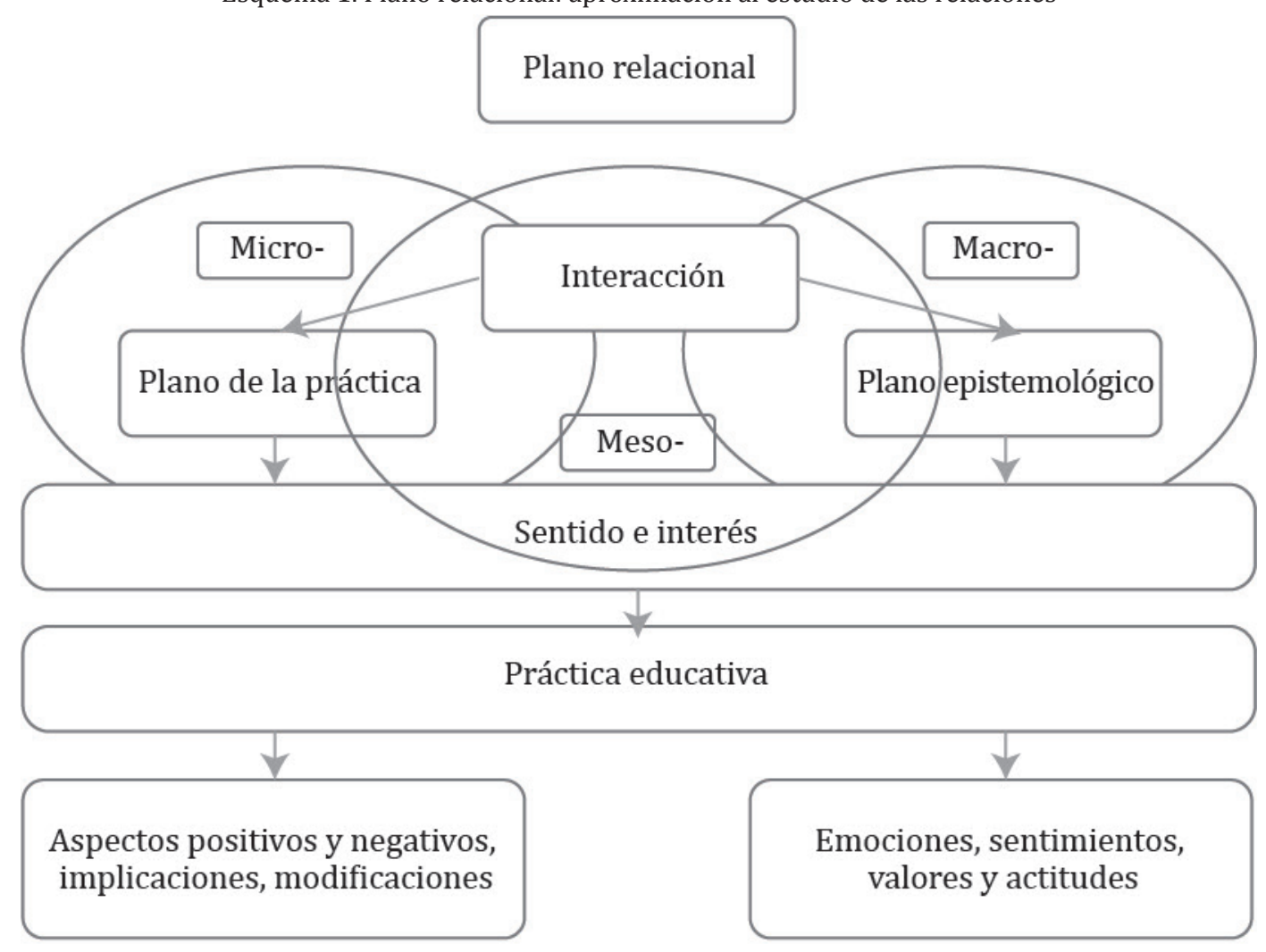

Fuente: Elaboración propia.

\section{DISCAPACIDAD, EDUCACIÓN Y DERECHOS HUMANOS: UNA CUESTIÓN RELACIONAL}

Si la educación inclusiva tiene que ver con el juego de las identidades que se establecen desde las mismas prácticas, cabe formular la pregunta por la identidad de los estudiantes con discapacidad. Es bastante conocida la historia que se ha señalado desde los estudios en torno a ella (Palacios, 2008; Brogna, 2009; Ferreira, 2008; Toboso y Arnau, 2008). Modelos y visiones que han hecho presencia a lo largo del devenir y que todavía conviven en diversos espacios hoy. La cuestión de la historia es también un asunto epistemológico y relacional, además de identitario, puesto que las experiencias cuentan no solo hechos, sino asignaciones de sentido en torno a lo que es, o no, una discapacidad. Identidades que juegan entre los sujetos de caridad, los enfermos, ciudadanos de segunda o sujetos de derechos.

La historia de la discapacidad está relacionada con su propia historia educativa; las visiones o representaciones corrieron a la par que las formas de proporcionales educación; por ello, es posible ubicar desde el recorrido educativo el estado que guarda el discurso que los define: pasaron de ser un problema personal a uno público (Borsay, 2008); un asunto médico a una cuestión de justicia social (Nussbaum, 2012); un cambio desde la propia mirada trágica hacia una social y de derecho (Oliver, 1998).

Este cambio puede ser visibilizado también desde los planos jurídico-normativos, con la aparición, por ejemplo, de la Declaración de los Derechos de las Personas con Retardo Mental (1971), la Declaración de los Derechos de los Impedidos (1975), 
el Plan de Acción Mundial para las Personas con Discapacidad (1982), las Normas Uniformes sobre la Igualdad de Oportunidades de las Personas con Discapacidad (1993), la Convención Americana para la Eliminación de Todas las Formas de Discriminación contra las Personas con Discapacidad (1999) y, de forma más reciente, la Convención sobre los Derechos de las Personas con Discapacidad (2008), que señala que la discapacidad no se encuentra en la persona, sino en la relación, es decir, aparece cuando se relaciona con otras personas o con un medio que le impiden participar con equidad e igualdad.

En la Convención sobre los Derechos de las Personas con Discapacidad (2008), el derecho a la educación es un derecho humano, y garantizarlo implica al Estado dar una educación con equidad e inclusión. La pregunta hasta este punto es ¿qué está pasando en las aulas con los estudiantes con discapacidad en su proceso inclusivo? ¿Los principios de justicia social, escolar y curricular (Torres, 2012) se han cumplido o por lo menos permean las prácticas educativas? ¿Qué tipo de prácticas están realizando los profesores para lograr la empresa inclusiva? Y sobre todo ¿qué características presentan las relaciones que se constituyen en dichas prácticas?

\section{PeRSPECTIVA METOdológica del eSTUdio}

El estudio es cualitativo de alcance descriptivo e interpretativo; analizó el aspecto relacional en los procesos de inclusión educativa de estudiantes con discapacidad desde la experiencia de los profesores. En este trabajo, utilizamos algunas herramientas del método de teoría fundamentada propuestas por Strauss y Corbin (2002), sobre todo los procesos de categorización y construcción teórica y conceptual. Las técnicas fueron el cuestionario cualitativo y el grupo focal. En este artículo solo presentamos los resultados del cuestionario cualitativo, el cual fue previamente piloteado.

En un primer momento, realizamos un ejercicio de microanálisis, es decir, una codificación línea por línea de los resultados del cuestionario. Fue un proceso de codificación abierta y posteriormente axial, lo cual llevó a un proceso de clasificación. Identificamos los códigos que encontramos a lo largo de las respuestas a las preguntas del cuestionario. Luego, agrupamos en busca de similitudes y diferencias para dar paso a la construcción de categorías. Ese instrumento se conformó de siete preguntas abiertas.

En la tabla 1 presentamos el objetivo del estudio y su alineación con las dimensiones y las preguntas construidas para el cuestionario cualitativo. Esta alineación parte del objetivo específico que está relacionado, de manera directa, con dos dimensiones de estudio, espacios a partir de los cuales se construyeron los ítems y las preguntas de los instrumentos, en este caso del cuestionario. Las dimensiones responden a las interrelaciones entre los tres sistemas abordados, el macro-, el meso- y el microsocial. Lo anterior ha sido cruzado con los principios de lo que aquí abordamos como "lo relacional", espacio donde se disponen valores, emociones, experiencias, tomas de postura, actitudes, etcétera, en torno a la acción realizada.

La lógica de construcción y analítica fue la siguiente: con base en uno de los objetivos del estudio (caracterizar las relaciones que se han construido a partir de la experiencia de la inclusión educativa de estudiantes con discapacidad desde la perspectiva de los profesores), buscamos el espacio entre los sistemas, es decir, aquellos 
elementos que podían hacer visible las relaciones entre micro- meso- y macro-. Un primer plano de encuentro relacional se puede percibir desde una cuestión práctica (micro- y meso-) (está en el plano de la práctica educativa). Aquí la indagación permitió hacer visible lo que acontecía tanto en el nivel áulico como el institucional.

En un segundo plano "epistemológico" (plano de los sistemas de creencias y significados), la atención se centró en las relaciones entre el sistema meso- y el macro-, cuyos elementos son más abstractos y tienen que ver con lo emocional, lo axiológico $y$, por ende, lo actitudinal. Cabe mencionar que lo relacional no es posible observarlo de forma directa; más bien el ejercicio se sujeta a la interpretación e inferencia a partir de los datos obtenidos; por ejemplo, cuando los profesores dan cuenta de alguna emoción o sentimiento, es posible pensar en un tipo de relación sostenida.

Tabla 1. Objetivos, dimensiones y preguntas

\begin{tabular}{|c|c|c|}
\hline Objetivo específico & Dimensión & Preguntas \\
\hline $\begin{array}{l}\text { Caracterizar las relaciones que se han } \\
\text { construido a partir de la experiencia } \\
\text { de la inclusión educativa de estu- } \\
\text { diantes con discapacidad desde la } \\
\text { perspectiva de los profesores }\end{array}$ & $\begin{array}{l}\text { Plano de la práctica: } \\
\text { relación micro- y mesosistémica }\end{array}$ & $\begin{array}{l}\text { 1. Implicaciones que el profesor con- } \\
\text { sidera que tiene que el trabajar con } \\
\text { un estudiante con discapacidad } \\
\text { 2. Los aspectos positivos de dicho } \\
\text { trabajo } \\
\text { 3. Los aspectos negativos de dicho } \\
\text { proceso y experiencia } \\
\text { 4. Las modificaciones que se recono- } \\
\text { ce se realizan en la práctica educativa } \\
\text { 5. Las emociones y los sentimientos } \\
\text { que se hacen presentes en el proceso } \\
\text { 6. Los valores que se despliegan o } \\
\text { manifiestan } \\
\text { 7. Las actitudes que se muestran en } \\
\text { cuanto a su inclusión }\end{array}$ \\
\hline
\end{tabular}

Contexto y participantes de estudio

El estudio se realizó con profesores de la ciudad de Puebla, en una universidad privada; se trató de profesionales que estaban cursando sus estudios de maestría en el área educativa en el verano de 2018. De un universo de 267 sujetos, fueron 41 participantes los que respondieron el cuestionario. El muestreo fue a conveniencia. La selección se basó en dos criterios de inclusión: que fueran profesores (1) que hayan atendido y trabajado desde su función de docencia con algún estudiante con discapacidad (2) sin importar su antigüedad frente a grupo, edad ni nivel educativo en el que se encontraran. Cabe mencionar que hubo un consentimiento informado sobre los alcances del estudio, y solicitamos su permiso y conocimiento en torno a la utilización de los datos. En este sentido, realizamos una codificación para garantizar el anonimato de los participantes. 
Tabla 2. Caracterización de los participantes

\begin{tabular}{|c|c|c|c|}
\hline Edad & Profesión/ocupación & Años de servicio & $\begin{array}{l}\text { Tipo de discapacidad } \\
\text { que ha atendido }\end{array}$ \\
\hline $\begin{array}{c}\text { Menos de } 24-5 \\
\text { participantes } \\
25-30-26 \text { partici- } \\
\text { pantes } \\
\text { 31-35 - } 6 \text { partici- } \\
\text { pantes } \\
\text { 36-40 - } 2 \text { partici- } \\
\text { pantes } \\
\text { 41-45 - } 0 \text { partici- } \\
\text { pantes } \\
\text { 46-50 - } 2 \text { partici- } \\
\text { pantes }\end{array}$ & $\begin{array}{c}\text { Psicólogo- } 6 \text { participantes } \\
\text { Profesor de preescolar- } 3 \text { participan- } \\
\text { tes } \\
\text { Profesor de educación especial- } 12 \\
\text { participantes } \\
\text { Profesor de inglés- } 1 \text { participante } \\
\text { Profesor de secundaria (normal)- } 10 \\
\text { participantes } \\
\text { Profesor de música- } 1 \text { participante } \\
\text { Profesor de primaria- } 4 \text { participantes } \\
\text { Profesor universitario- } \\
5 \text { participantes }\end{array}$ & $\begin{array}{l}\text { 1-5 - } 30 \text { participantes } \\
\text { 6-10 - } 8 \text { participantes } \\
\text { 11-15 - } 0 \text { participantes } \\
\text { 16-20 - } 1 \text { participante } \\
\text { 21-25- } 2 \text { participantes }\end{array}$ & $\begin{array}{c}\text { Intelectual - } 28 \text { estudian- } \\
\text { tes } \\
\text { Motriz - } 17 \text { estudiantes } \\
\text { Auditiva - } 16 \text { estudiantes } \\
\text { Visual - } 12 \text { estudiantes } \\
\text { Psicosocial - } 14 \text { estudian- } \\
\text { tes } \\
\text { Múltiple- } 3 \text { estudiantes } \\
\text { Lesión cerebral - } 1 \text { estu- } \\
\text { diante }\end{array}$ \\
\hline
\end{tabular}

\section{RESULTADOS}

Plano de la práctica: relación micro- y mesosistémica

En el esquema 2 presentamos la dimensión y las categorías construidas a partir de las preguntas del cuestionario. Debemos mencionar que lo aquí mostrado tiene que ver con un primer ejercicio de codificación y categorización que da cuenta de cuatro elementos que conforman la dimensión y sus respectivas categorías. 
Esquema 2. Dimensión: plano de la práctica: relación micro- y mesosistémica

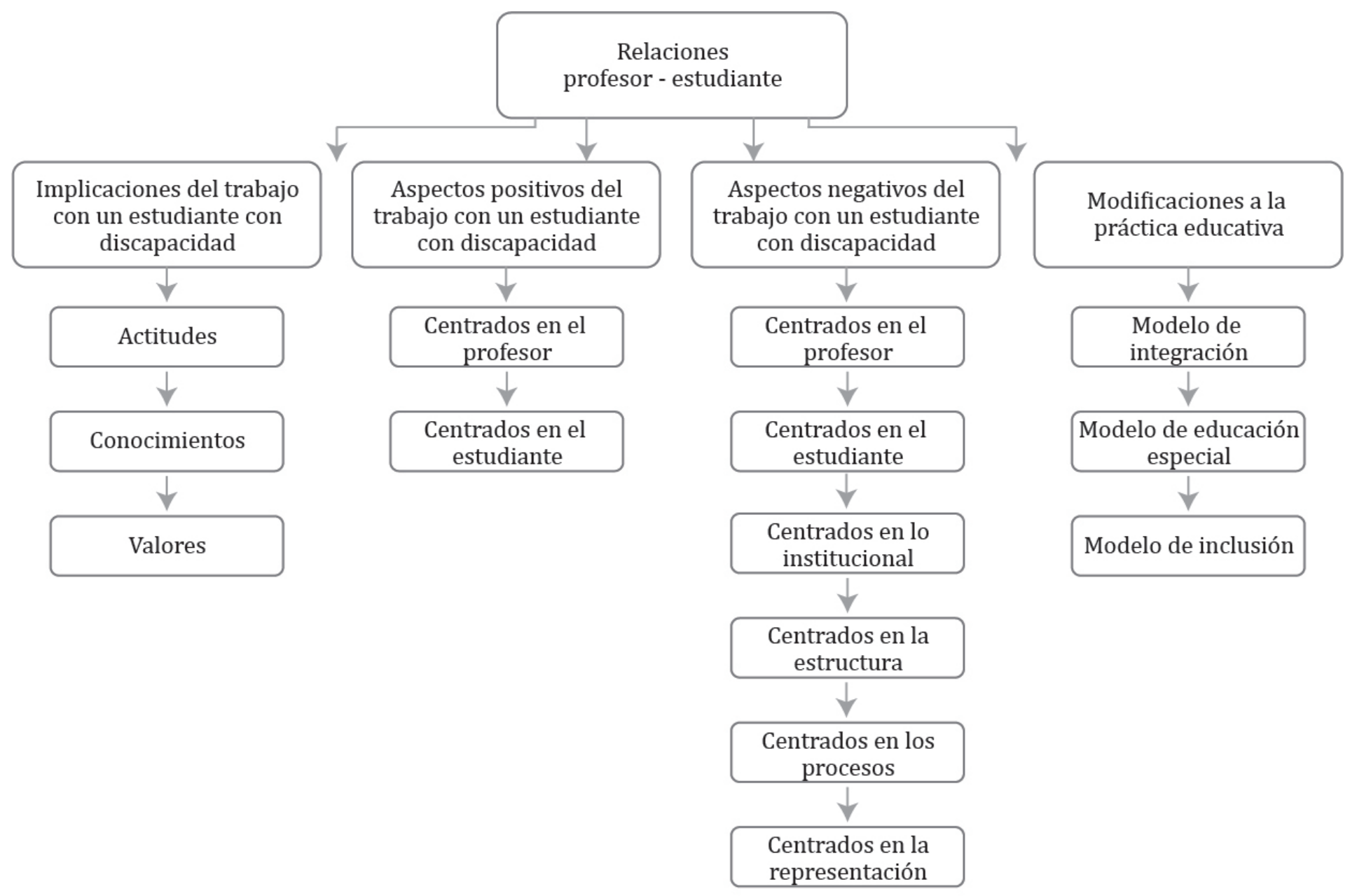

- Implicaciones del trabajo con un estudiante con discapacidad

Para la pregunta 1: "Trabajar con un alumno con discapacidad implica", identificamos 23 códigos iniciales, los cuales apuntaron sobre todo a cuestiones de capacitación, adecuaciones curriculares, necesidad de ser empático, y mostrar compromiso con su trabajo. En esta línea, tres categorías se construyeron a partir de los datos: conocimiento, actitud y valores. Como se observó en la tabla 2, las actitudes han sido señaladas por la mayoría de los participantes (trece veces); le siguen los valores (siete veces) $\mathrm{y}$ al final los conocimientos (tres veces). De acuerdo con el número de menciones, las actitudes siguen siendo el elemento central a rescatar con 42 menciones, seguido de los conocimientos, con 30 menciones en total, y los valores, con 23. 
Tabla 3. Implicaciones del trabajo con un estudiante con discapacidad

\begin{tabular}{|c|c|c|c|}
\hline \begin{tabular}{|c|} 
Ítems \\
$\begin{array}{l}\text { 1.Trabajar con un alumno } \\
\text { con discapacidad implica }\end{array}$
\end{tabular} & Códigos & Veces & Categorías \\
\hline \multirow{23}{*}{$\begin{array}{l}\text { 1.Trabajar con un alumno } \\
\text { con discapacidad implica }\end{array}$} & Sensibilización & 4 & Actitudes \\
\hline & Adecuaciones en la metodología & 16 & Conocimiento \\
\hline & Esfuerzo & 4 & Actitudes \\
\hline & Tolerancia & 7 & Actitudes \\
\hline & Inclusión & 3 & Valores \\
\hline & Capacitación/ conocimiento & 12 & Conocimiento \\
\hline & Creatividad & 2 & Conocimiento \\
\hline & Paciencia & 5 & Actitudes \\
\hline & Disposición & 4 & Actitudes \\
\hline & Respeto & 2 & Valores \\
\hline & Valor & 1 & Valores \\
\hline & Responsabilidad & 6 & Valores \\
\hline & Empatía & 8 & Actitudes \\
\hline & Buena actitud & 1 & Actitudes \\
\hline & Compromiso & 8 & Valores \\
\hline & Vocación & 3 & Actitudes \\
\hline & Cariño & 1 & Actitudes \\
\hline & Interés & 1 & Actitudes \\
\hline & Amor & 1 & Valores \\
\hline & Ética & 2 & Valores \\
\hline & Ser estricto & 1 & Actitudes \\
\hline & Ser más humano & 2 & Actitudes \\
\hline & Habilidades y actitudes & 2 & Actitudes \\
\hline
\end{tabular}

Para los profesores, existen varios elementos que se despliegan en el proceso de inclusión de un estudiante con discapacidad: por un lado, el profesor debe saber a quién va a tratar y cómo va a trabajar; este conocimiento implica la instauración de un diagnóstico que describa casi anticipadamente al otro y permita iniciar el proceso inclusivo. Si bien esto puede ser una cuestión inicial reconocida por los profesores, también da cuenta de su insuficiencia, pues dicho proceso necesita la conformación de actitudes hacia la tarea y la o las personas con las que interactuará, acciones que tienen fines en común sustentados en determinados valores para la puesta en marcha del proceso inclusivo. Para los profesores, la coexistencia de valores, conocimientos y actitudes son clave en todo proceso inclusivo; sin embargo, el conocimiento sobre el otro en el plano más "técnico" es central.

- Aspectos positivos del trabajo con un estudiante con discapacidad

En el caso de la pregunta 2: "Los aspectos positivos de trabajar con un estudiante con discapacidad son", identificamos tres categorías: centrados en el profesor, centrados en el alumno y centrados en el proceso. La primera aparece en dos códigos y tuvo 26 menciones; la segunda, solo en un código con 16 menciones; y la tercera también en un código y tuvo tres menciones. 
Tabla 4. Aspectos positivos del trabajo con un estudiante con discapacidad

\begin{tabular}{|c|c|c|c|}
\hline Ítems & Códigos & Veces & Categorías \\
\hline \multirow{4}{*}{$\begin{array}{l}\text { 2. Los aspectos positivos } \\
\text { de trabajar con un estu- } \\
\text { diante con discapacidad } \\
\text { son }\end{array}$} & Aprendizaje del profesor & 22 & Centrados en el profesor \\
\hline & Motivación & 4 & Centrados en el profesor \\
\hline & Inclusión & 3 & Centrados en el proceso \\
\hline & Aprendizaje de los estudiantes & 16 & Centrados en el estudiante \\
\hline
\end{tabular}

Algunos profesores reconocen que el proceso inclusivo no solo es para el alumno, sino que también para él (el profesor), pues se ve beneficiado al mejorar tanto su forma de ver la vida en general como su docencia. El proceso enriquece en lo personal y lo profesional; lo hace ser más humano e incrementar lo que sabe y ser más competente. Refieren que el estudiante con discapacidad y sus compañeros son favorecidos con ese proceso al tener la oportunidad de vivir experiencias que los sensibilizan.

Según los profesores, el proceso de inclusión beneficia al propio estudiante que tiene la oportunidad de socializar en un contexto diverso y a los demás compañeros al permitirles, mediante la convivencia, vivir la diversidad y trabajar aspectos axiológicos y de cuidado del otro, además de los contenidos escolares.

Por otra parte, los aspectos positivos no solo están posicionados en el profesor o en los estudiantes, no al menos de forma directa, sino lo que se potencializa tiene que ver con el propio proceso más amplio que sustenta la práctica inclusiva. Hay, entonces, un impacto social cuando se realiza una práctica de inclusión, el cual supera el espacio del aula y el escolar para permear a toda la estructura societal; en cierto sentido cabe, desde la perspectiva de los profesores, una función política del proceso inclusivo.

- Aspectos negativos del trabajo con un estudiante con discapacidad

La pregunta 3: "Los aspectos negativos de trabajar con un estudiante con discapacidad son", está representada en siete categorías: centrado en el estudiante, centrado en lo institucional, centrado en la estructura, centrado en la representación, centrado en los procesos, centrado en el profesor y centrado en los padres de familia. En la lista de códigos aparecen mayor número de veces: centrado en lo institucional (2), centrado en el profesor (2), centrado en la estructura (2) y centrado en la representación (2); sin embargo, de acuerdo con el número de menciones, podríamos colocar en un primer lugar la categoría B, con 16 menciones, seguida de la F, con 14; posteriormente, la G y la A, con diez menciones, la D, con siete, la C, con cinco y, por último, la E, con una mención. 
Tabla 5. Aspectos negativos del trabajo con un estudiante con discapacidad

\begin{tabular}{|c|c|c|c|}
\hline Ítems & Códigos & Veces & Categorías \\
\hline \multirow{12}{*}{$\begin{array}{l}\text { 3. Los aspectos negativos } \\
\text { de trabajar con un estu- } \\
\text { diante con discapacidad } \\
\text { son }\end{array}$} & Trabajo con padres de familia & 10 & Centrados en los padres de familia \\
\hline & $\begin{array}{l}\text { Falta de apoyo y conocimiento de } \\
\text { la escuela }\end{array}$ & 7 & Centrados en lo institucional \\
\hline & Estudiante por su discapacidad & 10 & Centrados en el estudiante \\
\hline & Falta de capacitación del profesor & 10 & Centrados en el profesor \\
\hline & Falta de recursos & 1 & Centrados en la estructura \\
\hline & Frustración & 2 & Centrados en los procesos \\
\hline & Intolerancia & 1 & Centrados en la representación \\
\hline & Desinterés & 1 & Centrados en los procesos \\
\hline & Más tiempo & 9 & Centrados en los institucional \\
\hline & Prejuicios & 6 & Centrados en la representación \\
\hline & Actitudes de otros profesores & 4 & Centrados en el profesor \\
\hline & Infraestructura & 4 & Centrados en la estructura \\
\hline
\end{tabular}

En la tabla 5 advertimos que todavía se percibe una visión esencialista y biomédica de la discapacidad, pues parece que el problema tiene que ver con la condición deficitaria del propio estudiante y que su avance o progreso depende de su discapacidad; por ende, de su capacidad para realizar las cosas. Sin embargo, hay señalamientos también a cuestiones contextuales, como lo es la propia institución escolar y su estructura.

En este sentido, los profesores reconocen el papel central que juega la institución para el logro del proceso inclusivo, y destacan los casi inexistentes apoyos desde la propia escuela para realizar adecuaciones u otras actividades relacionadas con la inclusión. También, los escasos espacios formativos para los profesores, los cuales deberían estar presentes cuando se ha aceptado el ingreso de un estudiante con discapacidad, pues implica construir las condiciones para que esa acción se dé con éxito. Aquí el llamado es al conocimiento especializado que se debe poseer para "atender" a dicho estudiante.

Del mismo modo, observamos un señalamiento a la propia forma de organización escolar, en la que el tiempo parece ser central para el logro de la empresa inclusiva. Una queja reiterada es que el profesor realiza su trabajo contra el tiempo que se ha dispuesto de manera homogénea, sin tomar en cuenta que una adaptación muchas veces indispensable tiene que ver con la flexibilidad temporal para que los estudiantes alcancen los objetivos. Lo anterior, aunado a la sobrepoblación de las aulas, espacios donde se debe atender una gran cantidad de estudiantes, lo cual hace más complejo el proceso inclusivo.

Otro elemento considerado importante es el entorno, el acceso a una infraestructura que sea adecuada y permita llevar a cabo los procesos inclusivos de aprendizaje y participación en la escuela. Sin embargo, no son las únicas barreras: los profesores reconocen que también hay las barreras simbólicas, es decir, el conjunto de creencias que muchas veces puede pesar más que la infraestructura y que siguen señalando a las personas con discapacidad como imposibilitadas o enfermos eternos.

En este plano de representación se hacen presentes las ideologías, los sistemas de razón, los prejuicios que en numerosas ocasiones imposibilitan incluso los intentos de efectuar una práctica educativa inclusiva. Cuando existe una representación desde la lógica esencialista y del modelo del déficit, esa condición parece estar 
en el centro de la práctica y, por tanto, constituirse en una barrera. En este punto, el problema radica en la forma en que se han pensado los procesos, la idea de un aprendizaje lineal, paulatino, rítmico y homogéneo, características clásicas de la organización escolar.

Otro aspecto reconocido como negativo es el papel de la familia en el proceso de inclusión. La queja de los profesores está en la percepción sobre el apoyo que les brindan los padres en el proceso, en cuanto a la falta de compromiso, la sobreprotección, el abandono y la escasa sensibilización que también los padres de los estudiantes con discapacidad muestran a la hora de tener que contribuir en el proceso de inclusión de sus hijos.

Por otra parte, para algunos profesores existe la idea de una cierta imposibilidad de educar en los mismos espacios a los estudiantes con discapacidad. Pareciera que el hecho de que el estudiante se encuentre en el aula implica un tratamiento individualizado que poco combina con el trabajo que el profesor debe realizar con todo el grupo. Así, la condición deficitaria prevalece desde una mirada esencialista de la discapacidad, es decir, centrada en su imposibilidad o falta, lo cual sigue resaltando una identidad deteriorada más que un reconocimiento de su diferencia. La idea de los profesores es que en el proceso de inclusión la respuesta debe ser un "simil" de la educación especial, en la cual se necesita un espacio único para el estudiante y una persona que lo atienda en el plano individual.

- Modificaciones a la práctica educativa

Respecto a la pregunta 4: "La práctica educativa de un docente se modifica con la presencia de estudiantes con discapacidad porque”, se asignaron tres categorías: modelo de educación especial, que aparece en la lista de códigos tres veces; modelo de integración, una vez; y el modelo de inclusión, cinco veces. En cuanto al número de menciones, el orden es el siguiente: aunque el modelo de integración aparece una sola vez, es el que mayor número de menciones tiene (22), seguido del modelo de inclusión, con 11 y el de educación especial, con 10. 
Tabla 6. Modificaciones a la práctica educativa

\begin{tabular}{|c|c|c|c|}
\hline Ítems & Códigos & Veces & Categorías \\
\hline \multirow{4}{*}{$\begin{array}{c}\text { 4. La práctica educativa } \\
\text { de un docente se modifica } \\
\text { con la presencia de estu- } \\
\text { diantes con discapacidad } \\
\text { porque }\end{array}$} & Realizas adecuaciones & 22 & Modelo de integración \\
\cline { 2 - 4 } & $\begin{array}{c}\text { Más atención al estudiante con } \\
\text { discapacidad }\end{array}$ & 8 & Modelo de educación especial \\
\cline { 2 - 4 } & Estudio y aprendizaje & 4 & Modelo de inclusión \\
\cline { 2 - 4 } & $\begin{array}{c}\text { Se modifica siempre no importa la } \\
\text { discapacidad }\end{array}$ & 3 & Modelo de inclusión \\
\cline { 2 - 4 } & Cambio de actitud & 2 & Modelo de inclusión \\
\cline { 2 - 4 } & $\begin{array}{c}\text { Conducta negativa del estudiante } \\
\text { con discapacidad }\end{array}$ & 1 & Modelo de educación especial \\
\cline { 2 - 4 } & $\begin{array}{c}\text { Desconocimiento por parte del } \\
\text { profesor }\end{array}$ & 1 & Modelo de educación especial \\
\cline { 2 - 4 } & Retos & 1 & Modelo de inclusión \\
\hline
\end{tabular}

Hay un aspecto señalado de manera reiterada: cuando existe un estudiante con discapacidad, es prioritario que se hagan adecuaciones y adaptaciones curriculares significativas, principio que se puede ubicar en el centro de la perspectiva integracionista, desde la cual se requiere la modificación de los contenidos y propósitos; este ajuste siempre ha de relacionarse con el tipo de discapacidad del estudiante (mirada esencialista).

No obstante, algunos profesores superan modelos de educación especial e integracionista, al no colocar al centro la condición deficitaria del estudiante con discapacidad; más bien, piensan en que todos deben participar por igual, que los "ajustes" no son para ese alumno en particular, sino para el colectivo, al reconocer la diversidad que desde antaño está presente en las aulas y escuelas.

\section{Plano epistemológico: relación macro- y mesosistémico}

La segunda dimensión tiene que ver con la relación entre lo macro- y mesosistémico, es decir, con el plano epistemológico. En el esquema 3 se muestran los resultados traducidos en categorías. 


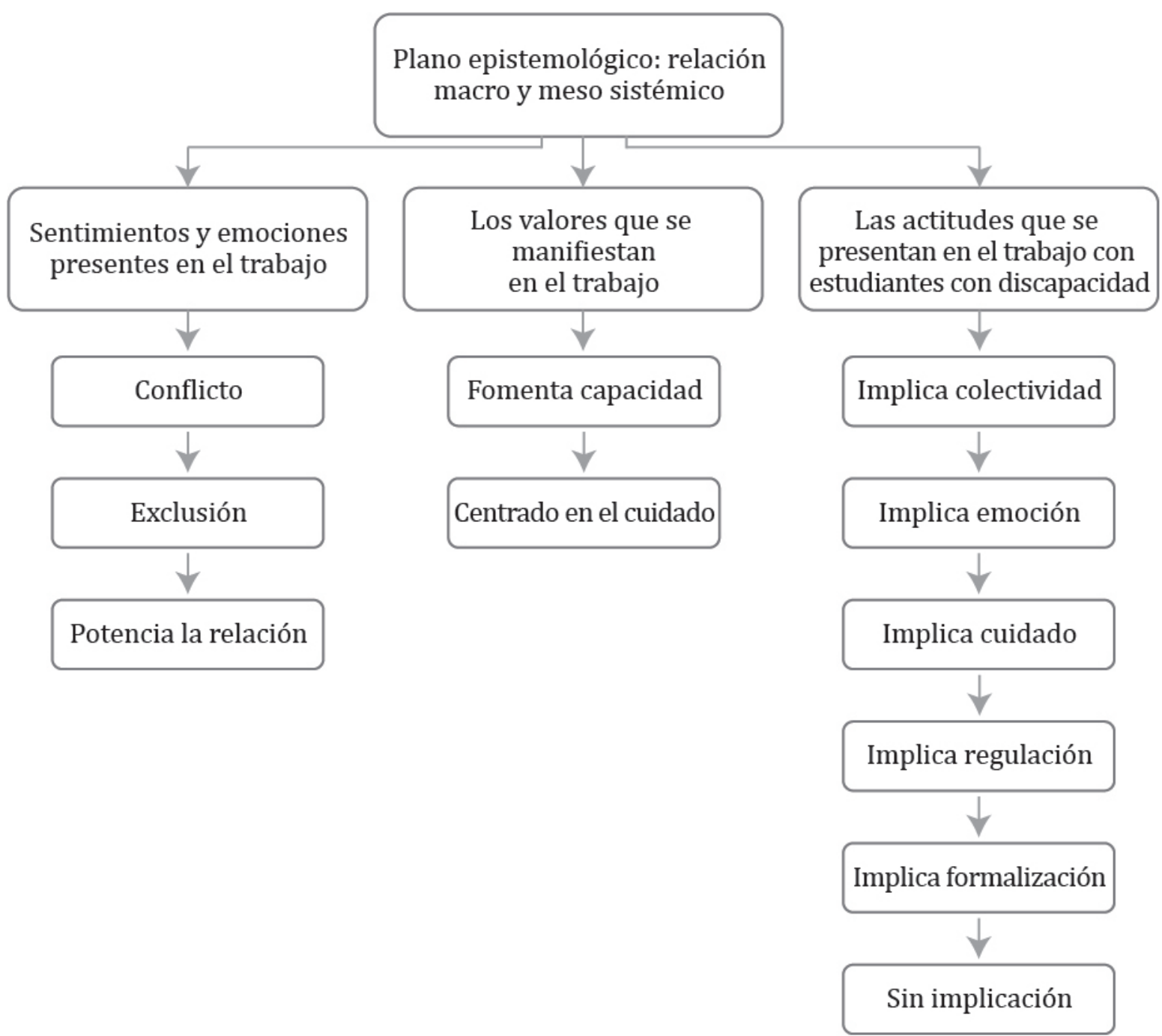

- Sentimientos y emociones en el trabajo

La pregunta 5: "Los sentimientos y emociones que se hacen presentes en los profesores cuando se trabaja con un estudiante con discapacidad son", tuvo también tres categorías: potencia la relación, conflicto y exclusión. La categoría A aparece en la lista de códigos en 13 ocasiones, la $\mathrm{B}$, en siete y la $\mathrm{C}$, en cuatro. En cuanto al número de menciones, la categoría A tuvo 84 menciones, seguida de la B, con 40 y la C, con ocho. 
Tabla 7. Sentimientos y emociones presentes en el trabajo

\begin{tabular}{|c|c|c|c|}
\hline Ítems & Códigos & Veces & Categorías \\
\hline \multirow{25}{*}{$\begin{array}{l}\text { 5. Los sentimientos y las } \\
\text { emociones que se hacen } \\
\text { presentes en los profe- } \\
\text { sores cuando se trabaja } \\
\text { con un estudiante con } \\
\text { discapacidad son }\end{array}$} & Amor/cariño/afecto & 13 & Potencia la relación \\
\hline & Frustración & 13 & Conflicto \\
\hline & Satisfacción & 7 & Potencia la relación \\
\hline & Alegría/felicidad & 15 & Potencia la relación \\
\hline & Tolerancia & 4 & Potencia la relación \\
\hline & Responsabilidad / compromiso & 4 & Potencia la relación \\
\hline & Incertidumbre/nerviosismo & 8 & Conflicto \\
\hline & Motivación & 2 & Potencia la relación \\
\hline & Temor/miedo & 9 & Conflicto \\
\hline & Emoción/entusiasmo & 10 & Potencia la relación \\
\hline & Respeto & 7 & Potencia la relación \\
\hline & Admiración/valoración & 4 & Potencia la relación \\
\hline & Sensibilidad & 2 & Potencia la relación \\
\hline & Paciencia & 4 & Potencia la relación \\
\hline & Empatía/comprensión & 8 & Potencia la relación \\
\hline & Irritabilidad/enojo & 5 & Conflicto \\
\hline & Positivos & 1 & Potencia la relación \\
\hline & Cansancio & 1 & Conflicto \\
\hline & Indiferencia & 1 & Exclusión \\
\hline & Compasión & 3 & Exclusión \\
\hline & Lástima & 2 & Exclusión \\
\hline & Tristeza & 3 & Conflicto \\
\hline & Esperanza & 3 & Potencia la relación \\
\hline & Impotencia & 1 & Conflicto \\
\hline & Rechazo & 2 & Exclusión \\
\hline
\end{tabular}

Las categorías fueron construidas pensando no tanto en que existen sentimientos y emociones positivas y negativas, sino en que, desde el espacio inclusivo, algunos de ellos pueden posibilitar el establecimiento de una relación entre el profesor y sus estudiantes, es decir, permiten que se dé la interacción y el encuentro, lo cual tiene como resultado el conocimiento mutuo y, por ende, el apoyo requerido. Desde esta lógica, están los sentimientos y emociones que muestran cierta preocupación por parte del profesor al surgir la incertidumbre en cuanto a su actuar; el temor de que no funcione lo realizado y, en ocasiones, un sentir de impotencia y frustración por aquello que desea, pero que no puede controlar o no ha logrado.

Los profesores dan cuenta, además, de emociones y sentimientos relacionados con el logro, la alta expectativa que tienen, y el reconocimiento del otro en su persona y particularidad. Precisamente, las relaciones implican algunos de estos componentes; es una apuesta a la posibilidad más que a la imposibilidad. Suponen la actitud favorable y la confianza en el otro y la apertura a identidades "diferentes", pero al final en paridad participativa.

Como observamos, este proceso "inclusivo" no es ajeno a ser conflictivo; sin embargo, cuando entramos en conflicto, algo está pasando desde nuestra subjetividad; hay una determinada crisis que refiere la puesta en tensión o el cuestionamiento reflexivo del propio profesor, elemento necesario o condición de posibilidad para la dinamización y el cambio en las prácticas educativas y los procesos de inclusión; es decir, el conflicto hace factible el cambio; así, que los profesores manifiesten temores, miedos, dudas o inseguridades no es más que parte del proceso y lo más probable es que lo superarán si no son indiferentes a esta situación. 
También se reconocen sentimientos y emociones que poco pueden propiciar el cambio educativo y mucho menos la relación; más bien rechazan, niegan, reducen o ignoran lo que está pasando, pues, en un primer momento, no existe reconocimiento del otro como un igual y como posibilitado para; más bien se recoge una mirada hacia la imposibilidad del logro educativo del propio estudiante con discapacidad.

- Valores que se manifiestan en el trabajo

En la pregunta 6: "Cuando un profesor trabaja con un estudiante con discapacidad se manifiestan valores como", se asignaron dos categorías: fomenta capacidad y centrado en el cuidado. La primera aparece relacionada con 11 códigos y la segunda, con 10 . En cuanto a las veces que fueron mencionadas por los participantes, la categoría $A, 114$ veces y la $B, 50$.

Tabla 8. Valores que se manifiestan en el trabajo

\begin{tabular}{|c|c|c|c|}
\hline Ítems & Códigos & Veces & Categorías \\
\hline \multirow{3}{*}{$\begin{array}{c}\text { 6. Cuando un profesor } \\
\text { trabaja con un estudiante } \\
\text { con discapacidad se mani- }\end{array}$} & Respeto & 35 & Fomenta capacidad \\
\cline { 2 - 4 } fiestan valores como & Igualdad & 4 & Fomenta capacidad \\
\cline { 2 - 4 } & Solidaridad & 19 & Fomenta capacidad \\
\cline { 2 - 4 } & Tolerancia & 19 & Centrado en el cuidado \\
\cline { 2 - 4 } & Responsabilidad/ compromiso & 25 & Fomenta capacidad \\
\cline { 2 - 4 } & Paciencia & 3 & Centrado en el cuidado \\
\cline { 2 - 4 } & Empatía & 18 & Fomenta capacidad \\
\cline { 2 - 4 } & Amor & 12 & Centrado en el cuidado \\
\cline { 2 - 4 } & Justicia & 3 & Fomenta capacidad \\
\cline { 2 - 4 } & Generosidad & 3 & Centrado en el cuidado \\
\cline { 2 - 4 } & Honestidad & 2 & Centrado en el cuidado \\
\cline { 2 - 4 } & Amistad & 4 & Fomenta capacidad \\
\cline { 2 - 4 } & Equidad & 1 & Fomenta capacidad \\
\cline { 2 - 4 } & Confianza & 2 & Fomenta capacidad \\
\cline { 2 - 4 } & Humildad & 2 & Centrado en el cuidado \\
\cline { 2 - 4 } & Lealtad & 1 & Fontrado en el cuidado \\
\cline { 2 - 4 } & Aceptación & 2 & Fomenta capacidad \\
\cline { 2 - 4 } & Valoración & 1 & Centrado en el cuidado \\
\cline { 2 - 4 } & Inclusión & 1 & Centrado en el cuidado \\
\hline
\end{tabular}

En el proceso de inclusión existen algunos valores que se relacionan más con procesos autónomos y otros que se encuentran más del lado de la ayuda que necesitan los estudiantes con discapacidad, es decir, un tipo de mirada caritativa. Por ejemplo, la categoría "fomenta la capacidad" tiene que ver con valores que están asociados a toda la teoría de la educación inclusiva, como la equidad, la justicia, la igualdad, el respeto y la propia inclusión. La categoría "centrado en el cuidado" da cuenta de valores como la tolerancia, paciencia, amor, aceptación, entre otros. Estos valores no pueden considerarse "inadecuados"; sin embargo, traducidos en acciones pueden representar retrocesos para el proceso inclusivo de los estudiantes con discapacidad, al mirarlos no como sujetos de derecho, sino como necesitados de asistencia y caridad. 
- Las actitudes que se presentan en el trabajo con estudiantes con discapacidad

En la pregunta 7: "Al trabajar con un estudiante con discapacidad, las actitudes que están presentes en el profesor son", se asignaron a los códigos siete categorías: implica colectividad, implica formación, implica emoción, implica cuidado, implica regulación, sin implicación e implica actividad. En cuanto a su relación con los códigos, la que mayor número de veces aparece es la categoría $\mathrm{A}$, con diez, seguida de la $\mathrm{B}$, con ocho, la C, con cinco, la E, con tres, la D y la G, con dos, y al final la F, con una vez. Respecto al número de veces en que aparecen, la categoría A tiene 47 menciones, la B, 20, la C, nueve, las D, E y G, cinco y la F, una.

Tabla 9. Las actitudes que se presentan en el trabajo con estudiantes con discapacidad

\begin{tabular}{|c|c|c|c|}
\hline Ítems & Códigos & Veces & Categorías \\
\hline \multirow{32}{*}{$\begin{array}{l}\text { 7. Al trabajar con un estu- } \\
\text { diante con discapacidad } \\
\text { las actitudes que están } \\
\text { presentes en el profesor } \\
\text { son }\end{array}$} & Servicio & 5 & Implica colectividad \\
\hline & Vocación & 1 & Implica formación \\
\hline & Entusiasmo & 4 & Implica emoción \\
\hline & Pasión & 1 & Implica emoción \\
\hline & Confianza & 4 & Implica formación \\
\hline & Comprensión & 1 & Implica formación \\
\hline & Disposición & 4 & Implica formación \\
\hline & Temor/incertidumbre & 2 & Implica emoción \\
\hline & Aprendizaje & 4 & Implica formación \\
\hline & Reflexión & 1 & Implica formación \\
\hline & Colaboración & 4 & Implica colectividad \\
\hline & Respeto & 7 & Implica colectividad \\
\hline & Amabilidad & 1 & Implica cuidado \\
\hline & Compromiso & 4 & Implica colectividad \\
\hline & Ayuda/apoyo & 7 & Implica colectividad \\
\hline & Paciencia & 3 & Implica regulación \\
\hline & Aceptación & 4 & Implica cuidado \\
\hline & Positiva & 8 & Implica colectividad \\
\hline & Negatividad & 1 & Implica emoción \\
\hline & Indiferencia & 1 & Sin implicación \\
\hline & Inclusión & 4 & Implica colectividad \\
\hline & Tolerancia & 1 & Implica regulación \\
\hline & Disponibilidad & 2 & Implica colectividad \\
\hline & Empatía & 5 & Implica colectividad \\
\hline & Desesperación & 1 & Implica emoción \\
\hline & Apertura & 3 & Implica formación \\
\hline & Creatividad & 2 & Implica formación \\
\hline & Autocontrol & 1 & Implica regulación \\
\hline & Responsabilidad & 4 & Implica colectividad \\
\hline & Proactividad & 2 & Implica actividad \\
\hline & Tenacidad & 3 & Implica actividad \\
\hline & Serenidad & 1 & Implica regulación \\
\hline
\end{tabular}

Las actitudes que señalaron algunos participantes fueron de colaboración, servicio, responsabilidad e inclusión, que pueden permitir o posibilitar el trabajo colectivo y comunitario, aspecto que es deseable en todo proceso inclusivo. En cambio, actitudes como la indiferencia no contribuyen a ningún tipo de implicación por parte del profesor que pretende trabajar la inclusión de un estudiante con discapacidad. 
De igual modo, los profesores reconocen actitudes que pueden relacionarse con la teoría de la educación inclusiva y las teorías del aprendizaje más socioeducativas: el servicio, la disposición de compañerismo son elementos sustanciales en todo proceso inclusivo cuya finalidad es la participación de todos en los espacios escolarizados. También se admite que el profesor se encuentra en un continuo proceso de aprendizaje; por tanto, la inclusión del estudiante con discapacidad puede ayudarlo a seguirse formando, aprendiendo nuevos modos de enseñanza, mejorando su docencia; en este sentido, no solo es el alumno el que se ve beneficiado, sino el propio profesor, siempre y cuando tenga esta actitud de apertura a conocer nuevas experiencias.

Por otra parte, las actitudes tienen que ver con formas de estar en donde las expectativas son altas, donde hay inquietud por parte del profesor, un estado anímico que lo impulsa al encuentro y el reto que implica el propio proceso inclusivo, lo desconocido, la duda, pero al final la reflexión de su actuar.

Los profesores refieren estados en los que se necesita trabajar desde la regulación personal y el cuidado para poder dar una respuesta pensada y analizada, la cual, en ocasiones, puede procurar una relación de cuidado, preocupación y atención por el otro.

Otras actitudes indicadas por los participantes señalan la movilización necesaria por parte del profesor, de la acción y práctica educativa, sustentada en un complejo proceso reflexivo y analítico, en el cual el compromiso, el ser constante y tenaz y ser proactivos puede contribuir al logro de la empresa inclusiva.

\section{CONCLUSIONES}

El encuentro de los profesores con los estudiantes con discapacidad parece ser una condición de posibilidad para que lo relacional se active; sin embargo, en ese proceso surgen diversas formas de relación, encuentros que refieren niveles macro-, meso- y micro-, y que es posible señalar con base en su experiencia. A continuación damos cuenta de la caracterización de las relaciones establecidas; si bien reconocemos que toda clasificación es arbitraria y que siempre hay traslapes e intercambios entre un tipo y otro, consideramos que para fines analíticos conviene comprometerse con los datos.

Antes incluso de pensar en unas formas de relación, es necesario que el estudiante llegue al aula, es decir, para que se dé la interacción y entren en conflicto las prácticas, el estudiante con discapacidad debe estar presente, pues en el encuentro cotidiano es posible que lo relacional se active y permita el conocimiento mutuo, el reconocimiento del otro como persona, esta condición de educabilidad que parece no darse si no hay encuentro.

En un primer momento podríamos afirmar que el conflicto que ocurre, en el cual los profesores señalan miedos y temores de diversa índole, parece funcionar como condición de posibilidad, pues detona procesos reflexivos posteriores; es decir, si bien los profesores mencionan que en el proceso convergen una serie de sentimientos y emociones, pueden permitir, a través de la reflexión, la relación situada, esto es, la comprensión particular del caso, al reconocer al estudiante con discapacidad desde su ser propio y no como una generalidad orgánica que comparte más de uno. 
La posibilidad del proceso inclusivo inicia entonces con un llamado al conflicto, espacio donde concurren elementos macro- (ordenamientos e imperativos sociales y morales), meso- (tensiones en la organización escolar) y micro- (cuestiones de aprendizaje y acceso al currículo). En este conflicto no se sabe qué va a pasar; la incertidumbre se apodera de las preocupaciones del profesor, pero, al hacerlo, en vez de representar un aspecto negativo, permite una determinada positividad, porque invita a la frustración y, por ende, a la conmiseración del otro, o bien, como lo han señalado algunos de los participantes, porque implica el reto que el docente necesita también para modificar su práctica educativa, que se ve potencializada con los procesos reflexivos que tiene el pensarla de otro modo, transformarla ahora sí situada en el estudiante y no en el propio profesor.

Con base en lo anterior, las relaciones se pueden pensar desde tres características: relaciones cuyo interés lo sustenta un sentido técnico; relaciones sustentadas en un interés con sentido práctico; y relaciones sustentadas con un interés con sentido transformador. El interés y sentido técnico refiere las relaciones cuya finalidad está dada de inicio desde lo que dicta el diagnóstico clínico y al margen de lo que puede, o no, aprender el estudiante. Estas relaciones buscan el aprendizaje de contenidos escolares como fin último, sin estar convencidos que los estudiantes con discapacidad puedan encontrarse en las aulas y aprender lo mismo que los demás.

Se defiende la idea que, para aprender, deben estar acompañados de una figura que los atienda en lo individual. La relación es superficial (técnica), pues no hay encuentro auténtico; las mediaciones suceden desde instrumentos (técnicos) como las adecuaciones que el "especialista" ha dispuesto que el profesor debe realizar y la información de lo que puede hacer, o no, el saber contenido en un diagnóstico también efectuado por otro profesional.

Con lo anterior no afirmamos que un diagnóstico no sea una herramienta útil e incluso necesaria; más bien es su contenido el que posibilita, o no, la relación que se puede iniciar. Por lo regular, muchos profesores, cuando hablan del diagnóstico, parecen aludir a las características de la propia discapacidad y, en ese sentido, al establecimiento de un tipo de nosografía que, en su fase anticipada y generalizada, induce a la construcción de algunos prejuicios sobre el estudiante, puesto que los diagnósticos, en general, resaltan las condiciones deficitarias que presentan las personas sin evidenciar la diversidad de situaciones, identidades y habilidades, las cuales diferencian a las personas que se ha decidido colocar bajo la etiqueta de discapacidad.

El diagnóstico más que apuntar la discapacidad, anticipadamente tendría que ser un instrumento que se hace junto con el estudiante con discapacidad, que señale las características y formas en que el estudiante aprende y, sobre todo, la naturaleza de los apoyos que requiere y con los cuales podrá participar sin que se precise, por lo menos en primera instancia, hacer grandes adecuaciones curriculares significativas. En este marco sería oportuno anticipar no la discapacidad en sí, sino los apoyos que serán básicos y fundamentales para recibir al estudiante y que se sienta a gusto y, principalmente, que pueda estar dando cuenta de su dignidad como persona.

Algunos profesores, cuando señalan la necesidad de especialistas, de personas que deben estar con el estudiante para que lo atiendan, promueven, tal vez sin querer, la inexistencia de un tipo de relación y conocimiento del estudiante, 
puesto que si este no es su responsabilidad, sí hay otro que puede trabajar con él; si ese que trabaja lo hace porque "sabe más" y porque fue capacitado para ello, la oportunidad de concretar un proceso auténticamente inclusivo corre peligro. Cuando los apoyos imposibilitan el encuentro y la relación, ya no son posibilitadores ni verdaderos apoyos, sino obstáculos para que el profesor nunca mire al estudiante con discapacidad en su humanidad, en sus posibilidades como persona, sin vencer la idea de estar hasta cierto punto "enfermo" de discapacidad, enfermedad que solo alguien que "sabe" puede curar.

Las relaciones sustentadas en un interés y sentido práctico apuntan más hacia las vivencias, las experiencias y situaciones que son posibles cuando se da la apertura a un estudiante con discapacidad en el aula regular; sin embargo, esos encuentros refieren un sentido común que atraviesa la práctica educativa, por ejemplo, visiones sobre los aprendizajes que el estudiante con discapacidad da a los demás como modelos de vida, de su fortaleza y esfuerzo, y que quedan como lección para quienes "son normales", ideas como "si ellos pueden, ustedes por qué no". Espacios donde se permite la presencia, pero que se agotan en la mera convivencia, sin que esto implique una auténtica participación. Esta acción impide pensar el encuentro identitario como posibilidad de reconocimiento cultural, de la diferencia, del otro que está relacionado desde su oportunidad de paridad y participación.

Con base en lo anterior, los procesos pueden darse sin "ningún problema", incluso basados en comentarios como "para mí no tiene discapacidad", pero, al no haberse permitido las tensiones, se entorpecen las acciones; los profesores que dicen no tener ningún conflicto, que todo parece no ser problemático, tampoco son realistas al negar las complejidades del proceso y la necesaria búsqueda constante de respuestas frente al reto impuesto. En concreto, la inclusión de un estudiante con discapacidad no está libre de ser problemático; negar esta condición es dudar de que la participación se esté dando más que la simulación.

Desde este interés y sentido práctico, los espacios escolares se convierten en lugares donde, por encima de constituir autonomía y capacidades en el estudiante, se ponga el cuidado y la asistencia, lección que se le da a los demás y que, sin embargo, se realiza con la finalidad de "ayudar" al otro, aunque esto represente una atención solo por su supuesta imposibilidad y no un reconocimiento como persona de derechos y con igual dignidad.

El interés y sentido transformador tiene que ver con las relaciones que reconocen las dificultades y también permiten espacios de reflexión y, sobre todo, de acción, y en las cuales los profesores admiten que la tarea no es fácil, pero una vez que el conflicto se ha dado y se han producido procesos reflexivos, se hace más fácil pensar la inclusión del otro, e identifica su aspecto procesual y temporal. Los cambios tal vez no suceden de la noche a la mañana, pero poco a poco se van haciendo visibles, no únicamente el aprendizaje de los propios estudiantes con discapacidad, sino las interacciones con los otros estudiantes y la aceptación del profesor sobre los aportes que el proceso inclusivo le ha traído a él.

En este sentido, el cambio visto como lección de vida para todos y como una oportunidad de seguir profesionalizándose. Desde este plano formativo, no es la información o el saber per se el que puede facilitar la inclusión de los estudiantes, sino la 
riqueza y potencialidad que tienen las relaciones que se establecen, en las cuales el diagnóstico no es la herramienta con base en la que gira el encuentro; es un instrumento entre muchos otros. Si se acepta que antes de ello está la riqueza del diálogo, la apertura a ver en el otro -más que una discapacidad- la construcción de una identidad que no es frágil, porque es parte de la condición humana de cada día.

En esta relación, el saber es importante, pero no traducido a cuánto se conoce de la discapacidad; más bien una práctica educativa que se pudiera denominar inclusiva y que le interese saber cómo realizar adecuaciones curriculares significativas y cómo se pueden construir espacios ricos en relaciones que permitan la participación de todos y la colegialidad como elementos que constantemente están "jalando" los aprendizajes, que son grupales e intersubjetivos más que individuales o personales.

El profesor que posee altas expectativas de logro contribuye a la constitución de un espacio dialógico; es capaz de entablar una comunicación con el estudiante no solo con la finalidad de "obtener" información "técnica”, sino con el propósito de ser más próximo y cercano, una relación que supera el nivel técnico-pedagógico que tanto se ha pensado especializado cuando se trata del trabajo o inclusión de personas con discapacidad.

\section{REFERENCIAS BIBLIOGRÁFICAS}

Adame, Emilia, Jacobo, Zardel y Alvarado, Julieta (2016). Sistema educativo e inclusión: más allá de la integración educativa. México: Trillas.

Adetoro, Rasheed (2014). Inclusive education in Nigeria -A mith or reality? Creative Education, vol. 5. pp. 1777-178. Recuperado de https://file.scirp. org/pdf/CE_2014111914214424.pdf

Ainscow, Mel (2014). Desarrollo de escuelas inclusivas. Ideas, propuestas y experiencias para mejorar las instituciones escolares. Madrid: Narcea.

Asamblea General de las Naciones Unidas (2000). Declaración del Milenio. Recuperado de https://documents-ddsny.un.org/doc/UNDOC/GEN/ N00/559/54/PDF/N0055954.pdf?OpenElement

Borsay, Anne (2008). ¿Problema personal o asunto público? Hacia un modelo de políticas para las personas con discapacidades físicas y mentales. En L. Barton (coord.). Superar las barreras de la discapacidad (pp. 171-189). España: Morata.

Brogna, Patricia (2009). Las representaciones de la discapacidad: la vigencia del pasado en las estructuras sociales presentes. En P. Brogna (comp.). Visiones y revisiones de la discapacidad (pp. 157-187). México: Fondo de Cultura Económica.

Chakraborti-Ghosh, Sumita (2017). Inclusive education in India: A developmental mileston from segregation to inclusión. Journal of Educational System, vol. 1, núm. 1. pp. 53-62. Recuperado de https://www.sryahwapublications. com/journal-of-educational-system/pdf/v1-i1/6.pdf

Contreras José, y Pérez de Lara, Nuria (2010). La experiencia y la investigación educativa. En J. Contreras y N. Pérez de Lara (comps.). Investigar la experiencia educativa (pp. 21-86). Madrid: Morata.

Cruz, Rodolfo (2013). Concepciones en torno al discurso de la integración educativa. Una mirada crítica, polisémica y deconstructiva. RIDE 10. Recuperado de ile:///C:/Users/RCRUZV/Downloads/134-520-1-PB\%20(2).pdf 
De la Vega, Eduardo (2010). Anormales, deficientes y especiales. Genealogía de la educación especial. Buenos Aires: Noveduc.

Douglas, Graeme, McLinden, Mike, Robertson, Christopher, Travers, Joseph \& Smith, Emma (2016). Including pupils with special educational needs and disability in national assessment: comparison of three country case studies through and inclusive assessment. International Journal of Disability, Development and Education, vol. 63, núm. 1, pp. 98-121. Recuperado de https:// www.dcu.ie/sites/default/files/carpe/Douglas\%20\%282015\%29.pdf

Echeita, Gerardo (2014). Educación para la inclusión o educación sin exclusiones. España: Nárcea.

Ferguson, Gail (2014). Including children with disabilities in Mainstream Education: A exploration of the challenges and considerations for parents and primary school teachers. Tesis de maestría. Recuperado de https://arrow.dit.ie/ cgi/viewcontent.cgi?article $=1076 \&$ context=aaschssldis

Ferreira, Miguel (2008). La construcción social de la discapacidad: habitus, estereotipos y exclusión social. Nómadas. Revista Critica de Ciencias Sociales y Jurídicas, vol. 17, núm. 1. pp. 221-232. Recuperado de https://revistas.ucm. es/index.php/NOMA/article/view/NOMA0808120221A/26443

Fitch, Frank (2003). Inclusión, exclusión and ideology: Special educations students changing sense of self. The Urban Review, vol. 35, núm 3, pp. 233-249.

Foucault, Michel (2005). El poder psiquiátrico. Curso en el Collège de France (1973-1974). Argentina: Fondo de Cultura Económica.

Foucault, Michel (1999). Los anormales. México: Fondo de Cultura Económica.

García, Ismael (2018). La educación inclusiva en la reforma educativa de México. Revista Nacional e Internacional de Educación Inclusiva, vol. 11, núm. 2, pp. 49-62. Recuperado de http://www.revistaeducacioninclusiva.es/index.php/ REI/article/view/373

Garro, Nuria (2017). Relación, razón relacional y reflexividad: tres conceptos fundamentales de la sociología relacional. Revista Mexicana de Sociología, vol. 79, núm. 3, pp. 633-600. Recuperado de http://www.scielo.org.mx/pdf/rms/ v79n3/0188-2503-rms-79-03-00633.pdf

Giné, Climent (2009). Aportaciones al concepto de inclusión. La posición de los organismos internacionales. En C. Giné (coord.), D. Durán, J. Font y E. Miquel. La educación inclusiva. De la exclusión a la plena participación de todo el alumnado (pp. 13-24). Barcelona: Cuadernos de Educación.

Goffman, Erving (2001). Estigma. La identidad deteriorada. Buenos Aires: Amorrortu.

Haug, Peder (2016). Understanding inclusive education: Ideals and reality. Scandinavian Journal of Disability Research, vol. 19. Recuperado de https:// www.tandfonline.com/doi/full/10.1080/15017419.2016.1224778?scroll=t op\&needAccess =true

Kiyuba, James \& Tukur, Sani (2014). Challenges of providing special education to children with disabilities: View of teachers and education officials. Tesis de licenciatura. University of Gavle Recuperado de http://www.diva-portal.org/ smash/get/diva2:764634/FULLTEXT01.pdf

López Melero, Miguel (2011). Barreras que impiden la escuela inclusiva y algunas estrategias para construir una escuela sin exclusiones. Revista Innovación Educativa, vol. 21, pp. 37-54. Recuperado de http://www.usc.es/revistas/index.php/ie/article/view/23 
Michailakis, Dimitris \& Reich, Wendelin (2009). Dilemas of inclusive education. Scienciedirect, vol. 3 Recuperado de https://www.researchgate.net/publication/251709328_Dilemmas_of_inclusive_education

Miles, Ssusie \& Singal, Nidhi (2008). The education for all and inclusive education debate: Conflict, contradiction or opportunity? International Journal of Inclusive Education. Recuperado de https://disability-studies.leeds.ac.uk/ wp-content/uploads/sites/40/library/miles-IJIE-MilesandSingal-resubmission.pdf

Mitchell, David (2015). Inclusive education is a multi- faceted concept. CEPS Journal, vol. 5, núm 1, pp. 9-30. Recuperado de https://www.pedocs.de/volltexte/2015/10611/pdf/cepsj_2015_1_Mitchell_Inclusive_Education.pdf

Mukherjee, Kaushik \& Bear, Sankara (2017). The challenges and opportunities to implement inclusive education in West Bengal. International Journal of $\mathrm{Hu}$ manities and Social Science Invention, vol. 6, núm. 12, pp. 38-43. Recuperado de http://www.ijhssi.org/papers/v6(12)/Version-2/F0612023843.pdf

Nussbaum, Martha (2012). Crear capacidades: propuesta para el desarrollo humano. Barcelona: Paidós.

Ngcobo, Jabulani \& Muthukrishna, Nithi (2011). The geographies of inclusión of students with disabilities in an ordinary school. South African Journal of Education, vol. 31, pp. 357-368. Recuperado de https://pdfs.semanticscholar. org/9b47/6630397c9fe8b737ae95bf6a6dbf631d53ab.pdf

Ocampo, Aldo (2015). Aproximaciones y descripciones generales sobre la formación del objeto de la educación inclusiva. En A. Ocampo (coord.). Los rumbos de la educación inclusiva en Latinoamérica en los inicios del siglo XXI. Cartografías para modernizar el enfoque (pp. 24-90). Santiago de Chile: CELEI.

Oliver, Mike (1998). ¿Una sociología de la discapacidad o una sociología discapacitada? En L. Barton (comp.). Discapacidad y sociedad (34-58). Madrid: Morata, SL.

Organización de los Estados Americanos (2001). Convención Americana para la Eliminación de Todas las Formas de Discriminación contra las Personas con Discapacidad. Recuperado de http://www.ordenjuridico.gob.mx/TratInt/ Derechos\%20Humanos/D8.pdf

Organización de las Naciones Unidas (ONU) (2008). La educación inclusiva: el camino hacia el futuro. Recuperado de http://www.ibe.unesco.org/ fileadmin/user_upload/Policy_Dialogue/48th_ICE/General_Presentation48CIE-4_Spanish_.pdf

ONU (2006). Convención sobre los Derechos de las Personas con Discapacidad. Recuperado de https://www.un.org/esa/socdev/enable/documents/tccconvs.pdf

ONU (1994). Conferencia Mundial sobre las Necesidades Educativas Especiales: Acceso y calidad. Recuperado de http://unesdoc.unesco.org/ images/0011/001107/110753so.pdf

ONU (1993). Normas Uniformes sobre la Igualdad de Oportunidades para las Personas con Discapacidad. Madrid: Editorial Centro Bibliográfico y Cultural La Coruña/Organización Nacional de Ciegos Españoles, ONCE.

ONU (1982). El decenio de acción mundial para las personas con discapacidad y el programa de acción mundial para las personas con discapacidad. Recuperado de http://www.un.org/spanish/esa/social/disabled/garesol.htm 
ONU (1975). Declaración de los Derechos de los Impedidos. Recuperado de http:// www.cinu.org.mx/temas/desarrollo/dessocial/integracion/ares_3477xxx.htm

ONU (1971). Declaración de los Derechos del Retrasado Mental. Recuperado de http://www.un.org/spanish/esa/social/disabled/dis50y40.htm

Organización de las Naciones Unidas para la Educación, la Ciencia y la Cultura (Unesco) (2015). Declaración de Icheon y Marco de Acción. Recuperado de http://www.unesco.org/new/fileadmin/MULTIMEDIA/FIELD/Santiago/ pdf/ESP-Marco-de-Accion-E2030-aprobado.pdf

Unesco (2004). Temario abierto sobre educación inclusiva. Recuperado de https://unesdoc.unesco.org/ark:/48223/pf0000125237_spa

Unesco (2001). Declaración de Cochabamba y recomendaciones sobre políticas educativas al inicio del siglo XXI. Recuperado de http://www.educacionespecial.sep.gob.mx/pdf/doctos/3Internacionales/12Declaraci on_Cochabamba.pdf

Unesco (2000). Foro Mundial sobre la Educación. Informe final. Recuperado de http://unesdoc.unesco.org/images/0012/001211/121117s.pdf

Unesco (1990). Declaración Mundial sobre Educación para Todos y Marco de Acción para Satisfacer las Necesidades Básicas de Aprendizaje. Recuperado de http://unesdoc.unesco.org/images/0012/001275/127583s.pdf

Orón, Víctor (2018). Educación centrada en el crecimiento de la relación interpersonal. Revista Studia Poliana, núm. 20, pp. 241-262. Recuperado de https://www.researchgate.net/publication/323739379_Educacion_centrada_en_el_crecimiento_de_la_relacion_interpersonal

Palacios, Agustina (2008). El modelo social de discapacidad: orígenes caracterización y plasmación en la Convención Internacional sobre los Derechos de las Personas con Discapacidad. México: Ed. Cinca.

Pié, Asun (2015). Por una corporeidad postmoderna. Nuevos tránsitos sociales y educativos para la interdependencia. España: UOC.

Popkewitz, Thomas y Brennan, Marie (2000). Reestructuración de la teoría social y política en la educación: Foucault y una epistemología social de las prácticas escolares. En T. S. Popkewitz y M. Brennan (comp.). El desafío de Foucault. Discurso, conocimiento y poder en la educación (pp. 17-48). Barcelona: Ediciones Pomares-Corredor.

Slee, Roger. (2012). La escuela extraordinaria. España: Ediciones Morata.

Skrtic, Thomas. (1996). La crisis en el conocimiento de la educación especial: una perspectiva sobre la perspectiva. En B. Franklin (comp.). Interpretación de la discapacidad. Teoría e historia de la educación especial (pp. 35-72). España: Ediciones Pomares.

Stainback, Susan y Stainback, William (2011). Aulas inclusivas. Un nuevo modo de enfocar y vivir el currículo. Madrid: Narcea.

Strauss, Anselm y Corbin, Juliet (2002). Bases de la investigación cualitativa. Técnicas y procedimientos para desarrollar la teoría fundamentada. Colombia: Universidad de Antioquia.

Toboso, Mario y Arnau, María (2008). La discapacidad dentro del enfoque de capacidades y funcionamientos de Amartya Sen. Revista Iberoamericana de Filosofía, Política y Humanidades, vol. 10, núm. 20, pp. 64-94. Recuperado de http://www.redalyc.org/articulo.oa?id=28212043004 
Thomas, Gary y Loxley, Andrew (2007). Deconstrucción de la educación especial y construcción de la inclusiva. Madrid: La Muralla.

Torres, Jurjo (2011). La justicia curricular. El caballo de Troya de la cultura escolar. Madrid, España: Morata.

Varela, Julia y Álvarez, Fernando (1989). Sujetos frágiles. Ensayos de sociología de la desviación. México: Fondo de Cultura Económica.

Vlachou, Anastasia (2007). Caminos hacia una educación inclusiva. Madrid: La Muralla. 